\title{
A New Indeterminacy Decision Making Approach Considering Partial Environmental State Knowledge
}

\author{
Bo FENG ${ }^{\text {a, }}$, Zhipeng HUI ${ }^{\mathrm{a}}$, Junwen FENG ${ }^{\text {a,b, } 1}$ \\ ${ }^{\mathrm{a}}$ Nanjing University of Science and Technology, 210094, China \\ ${ }^{\mathrm{b}}$ Nanjing Audit University Jinshen College, 210023, China
}

\begin{abstract}
The types of decisions people make depend on how much knowledge or information they have about the decision environmental situation or called state. There are three decision-making environments: decision making under certainty, decision making under uncertainty and decision making under risk. Based on possibility theory and evidence theory, a new indeterminacy decision making approach with the decision-maker's environmental knowledge information about the state of nature is proposed. This approach provides a general framework for three types of decision problems, that is, deterministic, risky and uncertain problems.
\end{abstract}

Keywords. Decision analysis, Possibility theory, Evidence theory, Fuzzy analysis

\section{Introduction}

Decision analysis can be used to develop an optimal strategy when a decision maker is faced with several decision alternatives and an uncertain or risk-filled pattern of future environmental state events. Even when a careful decision analysis has been conducted, the uncertain future events make the final consequence uncertain [1]. In some cases, the selected decision alternative may provide good or excellent results. In other cases, a relative unlikely future event may occur, causing the selected decision alternative to provide only fair or even poor results [2]. We begin the study of decision analysis by considering problems that involve reasonably few decision alternatives and reasonably few possible future environmental state events [3]. The payoff tables are introduced to provide a structure for the decision problem and to illustrate the fundamentals of decision analysis [4].

Consider the decision making problem expressed by Table 1:

Table 1 Decision making problem

\begin{tabular}{|l|cccc|}
\hline Alternatives & $S_{1}$ & $S_{2}$ & $\cdots$ & $S_{n}$ \\
\hline$A_{1}$ & $Q_{11}$ & $Q_{12}$ & $\cdots$ & $Q_{1 n}$ \\
$A_{2}$ & $Q_{21}$ & $Q_{22}$ & $\cdots$ & $Q_{2 n}$ \\
$\vdots$ & $\vdots$ & $\vdots$ & $\cdots$ & $\vdots$ \\
$A_{m}$ & $Q_{m 1}$ & $Q_{m 2}$ & $\cdots$ & $Q_{m n}$ \\
\hline
\end{tabular}

${ }^{1}$ Corresponding Author, Nanjing University of Science and Technology, Nanjing, China; E-mail: 313472714@qq.com 
Among the table, $\mathrm{A}=\{\mathrm{A} 1, \mathrm{~A} 2, \ldots, \mathrm{Am}\}$ is the unit of decision makings, they are independent and can not be substituted each other. $S=\left\{S_{1}, S_{2}, \cdots, S_{n}\right\}$ is a collection of various decision-making uncertain environmental situation which may called the state of nature. $Q_{i}$ means that the loss value of $A_{i}$ when decision-makers encounter the natural state $S_{j}$ (we suppose it is better for decision-makers when the loss value is larger in this paper). The question is which option is best for decision-maker?

If decision-makers know the state \{for example $S_{k}$ \} will happen, the question turns to a determined decision-making problem. The best option $A^{*}$ satisfies:

$$
E\left(A_{i} / S\right\}=Q_{i k} \quad E\left(A^{*} / S\right)=\max _{i} E\left(A_{i} / S\right)
$$

If decision-makers know probability distribution of natural state $P=\left\{P_{1}, P_{2}, \cdots, P_{m}\right\}$, where $P_{j}$ is the probability of occurrence of the natural state $S_{j}$ the problem turns to a risk-based decision making problem. At this time, decision-making choose the optimal option through the principle of the maximum expected loss value. The best option $A^{*}$ should satisfies:

$$
E\left(A_{i} / S\right)=\sum_{i, j=1}^{n} Q_{i j} P_{j}, \quad E\left(A^{*} / S\right)=\max _{i} E\left(A_{i} / S\right)
$$

If the decision-makers do not know the occurring of natural state, the question turns to a uncertain decision-making problem. Decision-makers must resort to certain criteria to make the appropriate decisions. There are five common decision criteria, they are, maximin criteria, maximax criteria, minimal regret criteria, Hurwicz criteria and equal opportunity criteria [1]. The reference [5] gives an optimism factor making-decision criteria through the five criterion above. We suppose the level of one decision-maker's optimism factor is $\alpha=\left(\alpha_{1}, \alpha_{2}, \cdots, \alpha_{n}\right)$, the best option $A^{*}$ based on optimism factor making-decision criteria should satisfies:

$$
E\left(A_{i} / S\right)=\alpha^{T} \underset{j}{\operatorname{Maxorder}}\left\{Q_{i j}\right\}, E\left(A^{*} / S\right)=\max _{i} E\left(A_{i} / S\right)
$$

Among it, Maxorder $\left\{Q_{i j}\right\}$ is the descending ordered vector of $Q_{i 1}, Q_{i 2}, \cdots, Q_{i n}, \alpha^{T} \beta$ is the vector product. Here $\alpha$ reflects the attitude of decision-makers. The determination of $\alpha$ can request Reference 3 .

In all the cases above, we call $E\left(A_{i} / S\right)$ is the evaluation value of decision $A_{i}$. Actually, the three cases above are all the extreme cases of such decision-making problems. The criteria to distinguish them is decision-maker' $\mathrm{s}$ evaluation of the state (or the state knowledge of decision-makers). There are various ways to express the state knowledge of decision makers actually [6]. The decision-makers should collect a lot of useful information when they evaluate the state and gradually reduce the scope of the estimates. Under normal circumstances, the evaluation of state is to figure out the probability of a state in the range of one space or the other. We can express this kind of state knowledge by possibility distribution or the structural reliability of evidence. We make the decision-makers' state knowledge as an evidence, that is to say, it's the proof which the state evaluation should be trusted. The decision-makers can form the confident degree of the state evaluation in the state space by these evidences. The degree is the reflection of the role of the state space, and it makes the estimation no longer blindness. 
In our paper, we will use Dempster-Shafter granule [7] to express the state knowledge of a decision-maker., and put the three decisions (determine type, risky type and uncertain type) in a unified framework.

\section{The expression of the state knowledge}

At first, we consider the following question. Suppose $\theta$ is a variable in a collection $\mathrm{X}, \mathrm{C}$ is a subset of $\mathrm{X}$. If the decision-maker only knows $\theta$ is an element of $\mathrm{C}$, we can use the possibility distribution function to express the knowledge of the decision-maker - " $\theta$ is an element of C", that is to say: $\pi_{\theta}(x)=\left\{\begin{array}{l}1, \text { if } x \in C \\ 0, \text { if } x \notin C\end{array}\right.$

Here, $\pi_{\theta}(x)$ is the possibility when $\theta$ equals to $\mathrm{x}$. Further, if $\mathrm{C}$ is a fuzzy set of $\mathrm{X}$, we can give the following equation: $\pi_{\theta}(x)=C(x)$

Among the $\mathrm{C}(\mathrm{x})$, we call $\mathrm{X}$ is the degree of membership in the fuzzy set $\mathrm{C}$. We call $\mathrm{C}(\mathrm{x})$ is the membership function of $\mathrm{C}$. The sense of $\mathrm{C}(\mathrm{x})$ is the possibility when $\theta$ equals to $\mathrm{x}$. We also suppose $\mathrm{m}$ is the map from the fuzzy set $\mathrm{x}$ to unit interval, that is to say, $m: I^{X \rightarrow}[0,1]$

Among it, $I^{X}$ is the collection of all the fuzzy set of $\mathrm{X}$. Suppose that $A_{i}, i=1,2, \cdots, p$ is a fuzzy subset of $\mathrm{X}, m\left(A_{i}\right)=a_{i} \neq 0$. Simultaneously, we call $A_{1}, A_{2}, \cdots, A_{p}$ are the central elements of $\mathrm{m}$ if it satisfies the following condition:

$$
\text { (1) } \sum_{i=1}^{p} m\left(A_{i}\right)=1 \quad \text { (2) } m(\phi)=0
$$

We call $\mathrm{m}$ is a function of supportive level. If $\theta$ is a variable in $\mathrm{X}$ and $\mathrm{m}$ is a function of supportive level, we call " $\theta$ is $\mathrm{m}$ " is a rule of $\mathrm{D}-\mathrm{S}$.

Now, let's consider $\theta$ is a variable expressed the state of decision-making, that is, $\theta$ is a variable which is defined in $S=\left\{S_{1}, S_{2}, \cdots, S_{n}\right\}$. We can use the D-S regulation to express the three types of decision-making statement.

Determined type, $\theta$ is $\mathrm{m}$ if $m\left(\left\{S_{k}\right\}\right)=1, S_{k} \in S$

Risky type, $\theta$ is $\mathrm{m}$ if $m\left(\left\{S_{j}\right\}\right)=P_{j}, j=1,2, \cdots, n$

Uncertain type, $\theta$ is $m$ if $m\{S\}=1$

Suppose B is a subset of S, if the possibility of the decision-makers' knowledge is $\theta$ at least $\alpha$, we can express the knowledge to " $\theta$ is $\mathrm{m}$ ", which $m(B)=\alpha$, $m(S)=1-\alpha$.

At this time, we can use the supportive function to express the common state knowledge. 


\section{The decision-making based on D-S rule}

Suppose $\theta$ is a variable which is defined in the state space $S=\left\{S_{1}, S_{2}, \cdots, S_{n}\right\}$. We can use the supportive function to express the knowledge of state space. Now let $E\left(A^{*} / m\right)$ be the evaluation when " $\theta$ is $\mathrm{m}$ ", at this time, $A$ " is the optimal choice under the state knowledge " $\theta$ is $m$ ". We can express it by, $E\left(A^{*} / m\right)=\max _{i} E\left(A_{i} / m\right)$

The question is how to estimate $E\left(A^{*} / m\right)$. Let' $\mathrm{s}$ discuss under the three circumstances below.

Case 1. Suppose $\mathrm{B}$ is the nonempty subset of $\mathrm{S}, \mathrm{m}$ is as follows: $m(B)=1, m(\phi)=0$

We use $\theta_{i}(B)$ to express the subset of possible loss under the Program Ai, that is $Q_{i}(B)=\left\{Q_{i j}: S_{j} \in B\right\}$. We can believe the evaluation function $E\left(A^{*} / \mathrm{m}\right)$ is based on $\theta_{i}(B)$. If $\mathrm{B}$ is a collection many elements, the decision-makers should choose a rule to pick up programs. Suppose the makers choose the decision by levels of optimism factor criteria.

The evaluation function can be expressed to:

$$
E\left(A^{*} / m\right)=\alpha^{T} \text { Maxorder } Q \quad{ }_{i}(B)
$$

In the equation, Maxorder $Q{ }_{i}(B)$ is the descending order of $Q_{i}(B)$, the dimension is same with B. $\alpha$ is the level of optimism factor.

Case 2. Suppose that B is a fuzzy set of $\mathrm{S}, \mathrm{m}$ is as follows: $m(B)=1, m(\phi)=0$

The possible loss under the decision Ai becomes the fuzzy set of loss,

$$
Q_{i}(B)=\bigcup_{j=1}^{n}\left(B\left(S_{j}\right) / Q_{i j}\right)=\left\{B\left(S_{1}\right) / Q_{i 1}, B\left(S_{2}\right) / Q_{i 2}, \cdots, B\left(S_{n}\right) / Q_{i n}\right\}
$$

Among that, $B\left(S_{j}\right)$ is the membership function of B under the $S_{j}$. In this case, we should decide an order by preparing the elements of $Q_{i}(B)$ when determine the $E\left(A^{*} / \mathrm{m}\right)$. Then make the decision by rule of level optimism factors. One possible way is to order by the membership, the other is ordering by the results of membership multiply loss value. Now, we decide the evaluation function by the level of fuzzy sets.

The definition $\beta$ of fuzzy set $Q_{i}{ }^{(\beta)}$ is: $Q_{i}{ }^{(\beta)}(B)=\left\{Q_{i j}: B\left(S_{j}\right) \geq \beta\right\}$

$Q_{i}{ }^{(\beta)}(B)$ may contains different elements for different $\beta \in[0,1]$, so the dimension of $Q_{i}^{(\beta)}(B)$ may be different, but none of them may be larger than the dimension of state space n.

Others, we suppose the same element of $Q_{i}{ }^{(\beta)}(B)$ may be different, the "same" means the numerically equal.

Suppose the optimism factor of decision-makers is $\alpha=\left(\alpha_{1}, \alpha_{2}, \cdots, \alpha_{n}\right)$, we may 
define the evaluation function of $\mathrm{Ai}, \quad E\left(A^{*} / m\right)$ is :

$$
E\left(A^{*} / m\right)=\int_{0}^{1}[\alpha]^{T} \text { Maxorder } Q_{i}{ }^{(\beta)}(B) d \beta
$$

The $[\alpha]$ here is the dimension of $\alpha=\left(\alpha_{1}, \alpha_{2}, \cdots, \alpha_{n}\right)$, Maxorder $Q \quad{ }_{i}^{(\beta)}$ is the descending ordered vector. If $\mathrm{n}=4$, the dimension of $Q_{i}{ }^{(\beta)}(B)$ is 3 , $[\alpha]=\left[\frac{\alpha_{1}}{\alpha_{1}+\alpha_{2}+\alpha_{3}}, \frac{\alpha_{2}}{\alpha_{1}+\alpha_{2}+\alpha_{3}}, \frac{\alpha_{3}}{\alpha_{1}+\alpha_{2}+\alpha_{3}}\right] ;$ if the dimension is 2, $[\alpha]=\left[\frac{\alpha_{1}}{\alpha_{1}+\alpha_{2}}, \frac{\alpha_{2}}{\alpha_{1}+\alpha_{2}}\right]$; if the dimension is $\mathrm{n},[\alpha]=\alpha$. So the $[\alpha]$ is changing by $\beta$. The evaluation function can be determined if the level optimism of factor $\alpha$ can be decided. Then we will give the application examples.

Case 3 (common case) $B_{k}, K=1,2, \cdots, p$ is the fuzzy set of $\mathrm{S}, \mathrm{m}$ is as follows:

$$
\left\{\begin{array}{c}
m\left(B_{K}\right)=\alpha_{K} \neq 0, K=1,2, \cdots, p \\
m(\phi)=0 \\
\sum_{K=1}^{p} m\left(B_{K}\right)=\sum_{K=1}^{p} \alpha_{K}=1
\end{array}\right.
$$

Here, we define the evaluation of $A_{i}$ is:

$$
E\left(A_{i} / m\right)=\sum_{K=1}^{p} E\left(A_{i} / m_{K}\right) \bullet \alpha_{K} \text { Where } m_{K}\left(B_{K}\right)=1, m_{K}(\phi)=0
$$

Notes: Case 1 and Case 2 are the special situation of Case 3. Further, if $B=\left\{S_{K}\right\}$, the case 1 becomes a determined type; if $\mathrm{B}=\mathrm{S}$, the case 1 becomes a certain type; if $B_{K}=\left\{S_{K}\right\}, K=1,2, \cdots, n, m\left(B_{K}\right)=P_{K}$, the case 3 becomes a risky type. So the case 3 is the unified structure of this paper. If contains a serious of expression of the state space knowledge.

\section{Numerical Examples}

Example 1. Consider a decision-making problem in Table 2

Table 2. Decision making example 1

\begin{tabular}{|l|lllll|}
\hline Alternatives & $S_{1}$ & $S_{2}$ & $S_{3}$ & $S_{4}$ \\
\hline $\mathrm{A}_{1}$ & 10 & -15 & 5 & 20 \\
$\mathrm{~A}_{2}$ & 5 & 5 & 10 & 10 \\
$\mathrm{~A}_{3}$ & 0 & 10 & 15 & 0 \\
\hline
\end{tabular}


Suppose the level optimism factor $\alpha=\left\{\alpha_{1}, \alpha_{2}, \alpha_{3}, \alpha_{4}\right\}=\{0.25,0.20,0.30$, $0.25\}$, the actual definition is, to every program, the weight of the decision-makers is $0.25,0.20,0.30$ and 0.25 . If the decision-maker only decide among the three programs, the corresponding optimism factor becomes

$$
[\alpha]=\left[\frac{\alpha_{1}}{\alpha_{1}+\alpha_{2}+\alpha_{3}}, \frac{\alpha_{2}}{\alpha_{1}+\alpha_{2}+\alpha_{3}}, \frac{\alpha_{3}}{\alpha_{1}+\alpha_{2}+\alpha_{3}}\right]=(0.33,0.27,0.40) ;
$$

if the decision-maker only decide among the two programs, the corresponding optimism factor becomes

$$
[\alpha]=\left[\frac{\alpha_{1}}{\alpha_{1}+\alpha_{2}}, \frac{\alpha_{2}}{\alpha_{1}+\alpha_{2}}\right]=(0.56,0.44) .
$$

In addition, the knowledge of decision-maker is “ $\theta$ is $\mathrm{m}$ ” $m(B)=1, m(\phi)=0$, $\mathrm{B}$ is the fuzzy set of $\mathrm{S}, \mathrm{B}=\left\{\frac{1}{S_{1}}, \frac{1}{S_{2}}, \frac{0.5}{S_{3}}, \frac{0.2}{S_{4}}\right\}$, now give the decision analysis.

Solutions:

At the moment,

$$
\begin{aligned}
& Q_{1}(B)=\left\{\frac{1}{10}, \frac{1}{-15}, \frac{0.5}{5}, \frac{0.2}{20}\right\} \\
& Q_{2}(B)=\left\{\frac{1}{5}, \frac{1}{5}, \frac{0.5}{10}, \frac{0.2}{10}\right\} \\
& Q_{3}(B)=\left\{\frac{1}{0}, \frac{1}{10}, \frac{0.5}{15}, \frac{0.2}{0}\right\}
\end{aligned}
$$

The corresponding sets are as follows in Table 3:

Table 3. Decision making Maxorder values

\begin{tabular}{|c|c|c|c|}
\hline$\beta$ & $\begin{array}{c}\text { Maxorder } \\
Q_{1}^{(\beta)}(B)\end{array}$ & $\begin{array}{c}\text { Maxorder } \\
Q_{2}^{(\beta)}(B)\end{array}$ & $\begin{array}{c}\text { Maxorder } \\
Q_{3}^{(\beta)}(B)\end{array}$ \\
\hline$[0,0.2]$ & $\{20,10,5,-15\}$ & $\{10,10,5,5\}$ & $\{15,10,0,0\}$ \\
\hline$[0.2,0.5]$ & $\{10,5,-15\}$ & $\{10,5,5\}$ & $\{15,10,0\}$ \\
\hline$[0.5,1.0]$ & $\{10,-15\}$ & $\{5,5\}$ & $\{10,0\}$ \\
\hline
\end{tabular}

$$
\begin{aligned}
& \text { So, } E\left(A_{1} / m\right)=\int_{0}^{1}[\alpha]^{T} \text { Maxorder } Q{ }_{1}^{\beta}(B) d \beta \\
& =\int_{0}^{0.2}\{0.25 \times 20+0.20 \times 10+0.30 \times 5+0.25 \times(-15)\} d \beta \\
& +\int_{0.2}^{0.5}\{0.33 \times 10+0.27 \times 5+0.40 \times(-15)\} d \beta \\
& +\int_{0.5}^{1}\{(0.56 \times 10+0.44 \times(-15)\} d \beta \\
& =4.75 \times 0.2+(-1.35) \times 0.3+(-1) \times 0.5=0.045 \\
& \quad E\left(A_{2} / m\right)=\int_{0}^{1}[\alpha]^{T} \text { Maxorder } Q_{2}^{\beta}(B) d \beta
\end{aligned}
$$




$$
\begin{aligned}
& =\int_{0}^{0.2}\{0.25 \times 10+0.20 \times 10+0.30 \times 5+0.25 \times 5\} d \beta \\
& +\quad \int_{0.2}^{0.5}\{0.33 \times 10+0.27 \times 5+0.40 \times 5\} d \beta \\
& \int_{0.5}^{1}\{(0.56 \times 5+0.44 \times 5\} d \beta \\
& =7.25 \times 0.2+6.65 \times 0.3+5 \times 0.5=5.945 \\
& E\left(A_{3} / m\right)=\int_{0}^{1}[\alpha]^{T} \text { Maxorder } Q_{3}^{\beta}(B) d \beta \\
& =\int_{0}^{0.2}\{0.25 \times 15+0.20 \times 10+0.30 \times 0+0.25 \times 0\} d \beta \\
& +\int_{0.2}^{0.5}\{0.33 \times 15+0.27 \times 10+0.40 \times 0\} d \beta \\
& +\int_{0.5}^{1}\{(0.56 \times 10+0.44 \times 0\} d \beta \\
& =5.75 \times 0.2+7.65 \times 0.3+5.6 \times 0.5=6.245
\end{aligned}
$$

Finally, the decision-maker should choose $A_{3}$.

Note: if $\alpha_{1}=1$, the other $\alpha_{n}=0$, at this point it corresponds to the optimistic decision; if $\alpha_{1}=1$, the other $\alpha_{n}=0$, Maxorder turns to be the increasing order and Minorder corresponds to the pessimistic decision. If $\alpha_{1}=\alpha^{\prime}, \alpha_{2}=1-\alpha$, Maxorder turns to be the composition of binary group with the maximum and minimum, and it corresponds to the optimistic decision; if turning the decision matrix to the regret matrix , and let $\alpha_{1}=1$, the other $\alpha_{n}=0$, increasing ordering, it corresponds to the regretted decision. If let $\alpha_{i}=\frac{1}{n}$ ( $\mathrm{n}=4$, in this example), $i=1,2, \cdots, n$, it corresponds to the equal possibility decision.

Example 2. The decision table is same as in example 1, but the knowledge of the decision-makers is following: $\mathrm{Q}$ is $\mathrm{m}, m\left(B_{1}\right)=0.7, m\left(B_{2}\right)=0.3, B_{1}=\left\{S_{1}, S_{2}\right\}$, $B_{2}=\left\{S_{1}, S_{2}, S_{3}, S_{4}\right\}$, the possibility when the evaluation of state by decision-makers is $B_{1}=\left\{S_{1}, S_{2}\right\}$ at least 0.7 . Others are all the same to example 1 .

Now, $Q_{1}\left(B_{1}\right)=\{0,-15\}, Q_{2}\left(B_{1}\right)=\{5,5\}, Q_{3}\left(B_{1}\right)=\{0,10\}$

So we has the equation:

$$
\begin{aligned}
& E\left(A_{1} / m_{1}\right)=0.56 \times 10+0.44 \times(-15)=-1 \\
& E\left(A_{2} / m_{1}\right)=0.56 \times 5+0.44 \times 5=5 \\
& E\left(A_{3} / m_{1}\right)=0.56 \times 10+0.44 \times 0=5.6
\end{aligned}
$$

Among the above equations: $m_{1}\left(B_{1}\right)=1, m_{1}(\phi)=0$

Similarly,

$$
Q_{1}\left(B_{2}\right)=\{10,-15,5,20\}, Q_{2}\left(B_{2}\right)=\{5,5,10,10\}, Q_{3}\left(B_{2}\right)=\{0,10,15,0\}
$$

So, $E\left(A_{1} / m_{2}\right)=0.25 \times 20+0.20 \times 10+0.30 \times 5+0.25 \times(-15)=4.75$

$$
\begin{gathered}
E\left(A_{2} / m_{2}\right)=0.25 \times 10+0.20 \times 10+0.30 \times 5+0.25 \times 5=7.25 \\
E\left(A_{3} / m_{2}\right)=0.25 \times 15+0.20 \times 10+0.30 \times 0+0.25 \times 0=5.75
\end{gathered}
$$

Among the above equations: $m_{2}\left(B_{2}\right)=1, m_{2}(\phi)=0$. So, 


$$
\begin{aligned}
& E\left(A_{1} / m\right)=0.7 \times(-1)+0.3 \times 4.75=0.725 \\
& E\left(A_{2} / m\right)=0.7 \times 5+0.3 \times 7.25=5.675 \\
& E\left(A_{3} / m\right)=0.7 \times 0.56+0.3 \times 5.75=5.645
\end{aligned}
$$

The decision-maker should choose the program $A_{1}$.

\section{Conclusion}

The paper is based on the decision analysis method by Possibility and Evidence theory. The method puts the common three decision type under a framework. And it used the knowledge which the decision-makers easily express. This approach is the generalization of the existed methods and has the decision-making supporting function.

The paper discussed the problem with discrete states and finite alternatives, which also can solve the infinite decision solutions through appropriate extension. Further, it is useful to extent the situation involving the multiple object decision-makings.

\section{References}

[1] David R. Anderson, Dennis J. Sweeney,Thomas A. Williams, Jeffrey D. Camm, James J. Cochran, Michael J. Fry, Jeffrey W. Ohlmann. An Introduction to Management Science: Quantitative Approaches to Decision Making, Fifteenth Edition, Cengage Learning, Inc. 2016.

[2] Kirkwood, C. W. An Overview of Methods for Applied Decision Analysis, Interfaces 22, 6 (NovemberDecember 1992): 28-39.

[3] Maxwell, Daniel T. Improving Hard Decisions, OR/MS Today 33, 6(December 2006): 51-61.

[4] Patchak, William M. "Software Survey: Decision Analysis." OR/MS Today 39, 5 (October 2012).

[5] Feng Junwen. Hierarchical Optimistic Approach for Uncertain Decision Making. Systems Engineering and Electronics, No.3, 1992

[6] Shafter G. A. Mathematical Theory of Evidence. Princeton University Press, Princeton, New Jersey, 1976

[7] Yager R. R. A General Approach to Decision Making with Evidential Knowledge in Uncertainty in Artificial Intelligence. Elsevier Science Publishers B. V. (North-Holland), 1986 